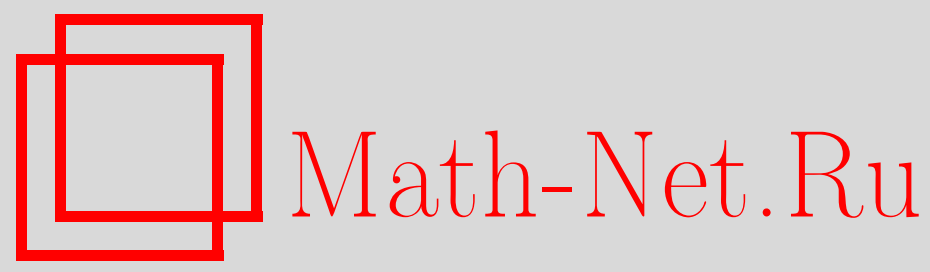

С. Зубе, Исключительные векторные расслоения на поверхностях Энриквеса, Матем. заметки, 1997, том 61, выпуск 6, 825-834

DOI: https://doi.org/10.4213/mzm1567

Использование Общероссийского математического портала Math-Net.Ru подразумевает, что вы прочитали и согласны с пользовательским соглашением http://www . mathnet.ru/rus/agreement

Параметры загрузки:

IP : 54.174 .149 .18

26 апреля 2023 г., $17: 45: 15$

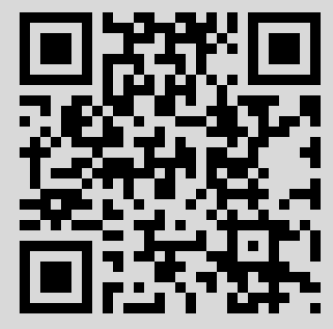




\title{
ИСКЛЮЧИТЕЛЬНЫЕ ВЕКТОРНЫЕ РАССЛОЕНИЯ НА ПОВЕРХНОСТЯХ ЭНРИКВЕСА
}

C. Зубе

\begin{abstract}
Основная цель настоящей статьи состоит в изучении исключительных векторных расслоений на поверхностях Энриквеса.

Библиограффия: 9 названий.
\end{abstract}

1. Введение. Исключительное расслоение $E$ на поверхности с иррегулярностью $q=h^{1}\left(O_{S}\right)$ и геометрическим родом $p_{g}=h^{2}\left(O_{S}\right)$ - это расслоение со следуюшими свойствами: $\operatorname{Ext}^{0}(E, E)=\mathbb{C}, \operatorname{Ext}^{1}(E, E)=q, \operatorname{Ext}^{2}(E, E)=p_{g}$. Ким и Най в [1]-[3] изучали на поверхностях Энриквеса экстремальные расслоения, которые по своим свойствам очень близки к исключительньм расслоениям. По определению экстремальное расслоение на поверхности Энриквеса - это жесткое расслоение $E$ такое, что $\operatorname{Ext}^{0}(E, E)=\mathbb{C}, \operatorname{Ext}^{1}(E, E)=0$, удовлетворяющее условию $\operatorname{Ext}^{2}(E, E)=\mathbb{C}$. Из теоремы Римана-Роха легко вытекает, что ранг любого исключительного расслоения нечетен, а любого экстремального - четен. В [2] Ким предложил характеризацию экстремальных расслоений на поверхностях Энриквеса. Такие расслоения существуют только на нодальных поверхностях и удовлетворяют условиям $c_{1}^{2}=4 n-2$, $c_{2}=n$ для $n>4$. У них есть и геометрический смысл. Оказьвается, существование экстремальных расслоений тесно связано с вложениями поверхности Энриквеса в грассманиан $G(2, n+1)$.

Основная цель работы - указать необходимые и достаточные условия существования исключительньх расслоений на поверхностях Энриквеса. Приводимое доказательство закрывает пробел, имеюшийся в доказательстве теоремы 4 из [2]. В последнем пункте мы также приводим конструкцию исключительных расслоений, основанную на некоторых конструкциях поверхностей Энриквеса и использующую модулярные операции (отражения). Отражение может оказаться чрезвычайно полезным при конструировании и изучении модулей пучков на поверхностях Энриквеса.

2. Поверхности Энриквеса. Гладкая неприводимая поверхность $S$ такая, что $h^{1}\left(O_{S}\right)=h^{2}\left(O_{S}\right)=0$ и $2 K_{S} \sim O_{S}$, назьвается поверхностью Энриквеса. Напомним, что дивизор $D$ на гладкой поверхности $X$ называется численно әффективным, если $D C \geqslant 0$ для всякой кривой $C$ на $X$. На протяжении всей статьи, зачастую без специального упоминания, будем использовать следующие полезные утверждения:

(А) $\left[4\right.$, следствие 3.1.3] Если $D$ - численно эффективньй дивизор и $D^{2}>0$, то $H^{1}\left(O_{S}(-D)\right)=0$ и $\chi(O(D))-1=\operatorname{dim}|D|=D^{2} / 2$. 
(Б) $[4$, предложение 3.1.4] Если у $|D|$ нет базисных компонент, то верно одно из следуюших утверждений:

i) $D^{2}>0$ и в $|D|$ существует неприводимая кривая $C$;

ii) $D^{2}=0$ и существует пучок $|P|$ рода 1 такой, что $D \sim k P$ для некоторого $k \geqslant 1$.

(В) $\left[4\right.$, гл. 4 , приложение, следствия 1 и 2] Если $D^{2} \geqslant 6$ и $D$ численно эффективный, то $D$ обилен, $2 D$ порож ден своими глобальными сечениями, $3 D$ очень обилен.

Поверхность Энриквеса $S$ назьвается нодальной (соответственно ненодальной), если в ней содержится (соответственно в ней отсутствует) гладкая (-2)-кривая. Поверхности Энриквеса почти всегда ненодальные. Всякая поверхность Энриквеса представляет собой эллиптическое слоение над $\mathbb{P}^{1}$, имеющее в точности два кратных слоя $F, F^{\prime}$ и эллиптический пучок $|2 F|=\left|2 F^{\prime}\right|$ с $K_{S}=F-F^{\prime}$. На общей поверхности Энриквеса имеется десять различных эллиптических пучков $\left|2 F_{1}\right|,\left|2 F_{2}\right|, \ldots,\left|2 F_{10}\right|$ (см. [4]).

3. Решетка Мукая. Дискретные инварианты пучков и расслоений на поверхностях типа К3 или на поверхности Энриквеса $X$ удобно представлять в виде векторов алгебраической решетки Мукая

$$
M(X)=H^{0}(X, \mathbb{Z}) \oplus \operatorname{Pic} X \oplus H^{4}(X, \mathbb{Z})=\mathbb{Z} \oplus \operatorname{Pic} X \oplus \mathbb{Z} \ni v=(r, D, s)
$$

со скалярным произведением $\langle\cdot, \cdot\rangle$

$$
\left\langle(r, D, s),\left(r^{\prime}, D^{\prime}, s^{\prime}\right)\right\rangle=r s^{\prime}+s^{\prime} r-D \cdot D^{\prime} .
$$

С каждым пучком $E$ на $X$ таким, что $c_{1}(E)=D, c_{2}(E) \in H^{4}(X, \mathbb{Z})=\mathbb{Z}$, ассоциируем вектор

$$
v(E)=\left(\operatorname{rank}(E), D, \frac{1}{2} D^{2}-c_{2}+\operatorname{rank}(E) \frac{\chi\left(O_{X}\right)}{2}\right),
$$

где $\chi\left(O_{X}\right)=1-q+p_{g}$, причем $\chi\left(O_{X}\right)=2$ для поверхности типа К 3 и $\chi\left(O_{X}\right)=1$ для поверхности Энриквеса. Из этой формулы и теоремы Римана-Роха вытекает равенство

$$
\begin{aligned}
& \langle v(F), v(E)\rangle=\langle v(E), v(F)\rangle=\chi(E, F) \\
& =\operatorname{dim} \operatorname{Ext}^{0}(E, F)-\operatorname{dim} \operatorname{Ext}^{1}(E, F)+\operatorname{dim} \operatorname{Ext}^{2}(E, F) .
\end{aligned}
$$

Из того, что $K_{X}$ численно равно 0, вытекает, что $\chi(E, F)=\chi(F, E)$ на поверхности типа К3 или поверхности Энриквеса. Для короткой точной последовательности $0 \rightarrow F \rightarrow$ $E \rightarrow G \rightarrow 0$ справедливы следуюшие равенства:

$$
v(E)=v(F)+v(G)=\left(r(F)+r(G), c_{1}(F)+c_{1}(G), s(F)+s(G)\right) .
$$

\section{4. Исключительные расслоения.}

ОПРЕДЕЛЕниЕ. Пучок $E$ на поверхности $S$ назьвается исключительным, если

$$
\operatorname{dim} \operatorname{Ext}^{0}(E, E)=1, \quad \operatorname{dim} \operatorname{Ext}^{1}(E, E)=q, \quad \operatorname{dim} \operatorname{Ext}^{2}(E, E)=p_{g} .
$$

Из теоремы Римана-Роха вытекает, что

$$
\chi(E, E)=r^{2} \chi\left(O_{S}\right)+(r-1) c_{1}^{2}-2 r c_{2} .
$$

Так как решетка $H^{2}(S, \mathbb{Z})$ четная и $\chi\left(O_{S}\right)=1$ для поверхности Энриквеса $S$, то ранг исключительного расслоения $E$ нечетен. Для описания исключительных векторных расслоений необходим следующий результат Кулешова. 
Tеорема 1 [5]. Пусть $X$ - гладкая поверхность типа К3, H - произвольный обильный дивизор на $X$ и $v=(r, D, s)(r>0)$ - исключительный вектор (т.е. $\left.v^{2}=2\right)$, принадлежащий решетке Мукая на X. Тогда существует простое $\mu_{H}$-полустабильное расслоение $E$, реализующее вектор $v($ m.e. $v=v(E))$.

Начнем с изложения нескольких простых фактов. Первый касается пучков без кручения, удовлетворяющих некоторому гомологическому условию.

ПРЕДЛОЖЕНИЕ 1. Пусть $E-$ пучок без кручения на гладкой поверхности $S$ и $\operatorname{dim} \operatorname{Ext}^{1}(E, E)=1$ или 0. Тогда $E$ локально свободен.

ДокАЗАТЕЛЬСтво. Имеет место следующая точная последовательность:

$$
0 \rightarrow E \rightarrow E^{* *} \rightarrow M \rightarrow 0
$$

где $E^{* *}$ - дважды двойственный пучок к $E$ и коядро $M$ имеет конечную длину. С помощью результата Мукая [6, следствия 2.11 и 2.12$]$ приходим к следующему неравенству:

$$
\operatorname{dim} \operatorname{Ext}^{1}\left(E^{* *}, E^{* *}\right)+\operatorname{dim} \operatorname{Ext}^{1}(M, M) \leqslant \operatorname{dim} \operatorname{Ext}^{1}(E, E) .
$$

В силу того, что $v^{2}(M)=0$, получаем $\operatorname{dim} \operatorname{Ext}^{1}(M, M)=2 \operatorname{dim} \operatorname{End}_{O_{S}}(M)$. Так как $\operatorname{Ext}^{1}(E, E)=0$ или 1 , то $M=0$ и $E=E^{* *}$. Поэтому $E$ локально свободен.

Теперь сформулируем основной результат.

Tеорема 2. Пусть $S$ - гладкая поверхность Энриквеса, $v=(r, D, s) \in M(S)$ $(r>0) u v^{2}=1$. Тогда

i) существует обильный дивизор $H$ такой, что у $D \cdot H$ и $r$ нет общего делителя, больиего единицы (т.е. $(D \cdot H, r)=1)$;

ii) для любого обильного дивизора $H$, удовлетворяющего условию $(D \cdot H, r)=1$, существует единственное $H$-стабильное исключительное векторное расслоение E такое, что $v(E)=v$.

ДокАЗАтельство. і) Так как $v^{2}=1$, то $2 r s-D^{2}=1$. Это означает, что $D \notin$ $r^{\prime} \cdot H^{2}(S, \mathbb{Z})$ для любого числа $r^{\prime}$ такого, что $\left(r^{\prime}, r\right)>1$ (здесь $(\cdot, \cdot)$ - наибольший общий делитель). Наша решетка $H^{2}(S, \mathbb{Z})$ унимодулярна, поэтому существует $X \in H^{2}(S, \mathbb{Z})$ такой, что $(X \cdot D, r)=1$. Пучок $H_{k}=X+k r H$ будет очень обильньм для любого обильного дивизора $H$ и $k \gg 0$. Очевидно, пучок $H_{k}$ удовлетворяет условию $\left(H_{k} \cdot D, r\right)=1$. Утверждение i) доказано.

ii) Обозначим через $X$ универсальное накрьвающее пространство для $S ; X$ является К3-поверхностью. Отображение накрытия обозначим через $\pi$. Рассмотрим вектор $\widehat{v}=\left(r, D^{\prime}, s^{\prime}\right)=\pi^{*}(v)$ на К3-поверхности $X$. Оказывается, что $\widehat{v}^{2}=2$, поэтому по теореме Кулешова на $X$ существует $\widehat{H}=\pi^{*}(H)$-полустабильное исключительное векторное расслоение $F$ такое, что $\widehat{v}(F)=\widehat{v}$. На самом деле, $F \widehat{H}$-стабильно. Действительно, $\left(\widehat{H} \cdot D^{\prime}, r\right)=(2 H \cdot D, r)=1$ (напомним, что число $r$ нечетно) и для произвольного подпучка $W$ такого, что $0<\operatorname{rank}(W)<\operatorname{rank}(F)$, будет справедливо неравенство

$$
\frac{c_{1}(W) \cdot \widehat{H}}{\operatorname{rank}(W)}<\frac{D^{\prime} \cdot \widehat{H}}{\operatorname{rank}(F)}
$$


Следовательно, такой пучок $F \hat{H}$-стабилен. Таким образом, оба пучка $F$ и $\sigma^{*}(F) \hat{H}$-стабильны, где $\sigma$-инволюция без неподвижных точек на $X$ такая, что $X / \sigma=S$. Легко видеть, что $\chi\left(F, \sigma^{*}(F)\right)=2$. Поэтому существует ненулевой гомоморфизм из $F$ в $\sigma^{*}(F)$, который должен быть изоморфизмом, так как оба векторных расслоения $\widehat{H}$-стабильны и имеют одинаковьй детерминант. Этот изоморфизм означает, что существует расслоение $E$ на поверхности $S$ такое, что $\pi^{*}(E)=F$. Конечно, векторное расслоение $E$ $H$-стабильно и $v(E)=v$. Предположим, что существует другое $H$-стабильное векторное расслоение $\widehat{E}$ такое, что $v(E)=v(\widehat{E})$. Тогда $\chi(E, \widehat{E})=1$, значит существует нетривиальное отображение $\varphi: E \rightarrow \widehat{E}$ или согласно принципу двойственности Серра $\rho: \widehat{E} \rightarrow E \otimes K$. Из предположения стабильности вытекает, что оба отображения должны быть изоморфизмами. Но тогда $E=\widehat{E}$ или $\operatorname{det} \rho$ задает ненулевой элемент в каноническом классе. Это противоречие доказьвает единственность $H$-стабильного расслоения.

Заметим, что из условия $\operatorname{Ext}^{1}(E, E)=0$ вытекает только то, что пространство модулей таких расслоений дискретно. Утверждение іi) означает, что такое $E$ единственно, и поэтому пространство модулей состоит только из одной точки.

ЗАмЕчАниЕ. Неизвестно, является ли $H$-стабильное исключительное векторное расслоение $E G$-нестабильным относительно любого другого обильного дивизора $G$. Из теоремы вытекает, что такое возможно только, если $\left(G \cdot c_{1}(E), \operatorname{rank}(E)\right)>1$.

5. Примеры. Приведем несколько конкретных примеров исключительных векторных расслоений. Один путь их построения, как в теореме 2 , состоит в том, чтобы найти стабильное инвариантное исключительноевекторное расслоение на К3-поверхности. Рассмотрим представление Хирокавы для поверхности Энриквеса (подробности см. в [7]). Введем на $\mathbb{P}^{3}$ координаты $\left(z_{0}: z_{1}: z_{2}: z_{3}\right)$, в которых квадрика $Q=\mathbb{P}^{1} \times \mathbb{P}^{1}$ вкладывается с помощью отображения

$$
z_{0}=x_{0} y_{0}, \quad z_{1}=x_{1} y_{1}, \quad z_{2}=x_{0} y_{1}, \quad z_{3}=x_{1} y_{0}
$$

Определим инволюцию $\tau$ на $\mathbb{P}^{3}$ формулой $\tau\left(z_{0}: z_{1}: z_{2}: z_{3}\right)=\left(z_{0}: z_{1}:-z_{2}:-z_{3}\right)$; тогда $Q$ инвариантна относительно действия $\tau$ и ограничение $\tau$ на $Q$ имеет вид

$$
\tau\left(x_{0}: x_{1}\right)\left(y_{0}: y_{1}\right)=\left(x_{0}:-x_{1}\right)\left(y_{0}:-y_{1}\right)
$$

Инволюция $\tau$ имеет на $Q$ четыре неподвижные точки

$$
\left(x_{0}: x_{1}\right)\left(y_{0}: y_{1}\right)=(1: 0)(1: 0),(1: 0)(0: 1),(0: 1)(0: 1),(0: 1)(1: 0) \text {. }
$$

Рассмотрим $\tau$-инвариантный многочлен бистепени $(4,4)$, определяющий $\tau$-инвариантную кривую $B$. Предположим, что на $B$ нет неподвижных точек инволюции $\tau$. Рассмотрим двукратное накрытие $X$ поверхности $Q$, разветвленное над $B$. Оказьвается, что $X$ является К3-поверхностью и инволюция $\tau$ индуцирует на $X$ инволюцию $\sigma$, не имеющую неподвижных точек. Таким образом, $X / \sigma=S$ является поверхностью Энриквеса. Заметим, что таким образом можно получить общую поверхность Энриквеса. Обозначим через $\pi: X \rightarrow S$ отображение факторизации, через $\varphi: X \rightarrow Q$ отображение двукратного накрытия, разветвленное над $B$. Если $E$ - исключительное векторное расслоение 
на $Q$, то расслоение $\varphi^{*}(E)=\widehat{E}$ также является исключительным. Действительно, согласно формуле проекции $H^{i}\left(\operatorname{End}_{O_{X}}(\widehat{E})\right)=H^{i}\left(\operatorname{End}_{O_{Q}}(E)\right) \oplus H^{i}\left(\operatorname{End}_{O_{Q}}(E) \otimes\left(-K_{Q}\right)\right)$ (напомним, что $B=-2 K_{Q}$ ). Так как в силу двойственности Серра

$$
H^{i}\left(\operatorname{End}_{O_{Q}}(E) \otimes\left(-K_{Q}\right)\right)=H^{2-i}\left(\operatorname{End}_{O_{Q}}(E)\right),
$$

то векторное расслоение $\widehat{E}$ на К3-поверхности $X$ является исключительным. Это расслоение $\sigma$-инвариантно, так как $E$-инвариантно на квадрике $Q$. (Действительно, расслоение $E$ жесткое, поэтому оно $\mathrm{PGL}(2) \times \mathrm{PGL}(2)$-однородно.) Ввиду того, что $\widehat{E}$ $\sigma$-инвариантно, существует векторное расслоение $F$ на $S$ такое, что $\widehat{E}=\pi^{*}(F)$. Легко видеть, что $v^{2}(F)=1$ и $F$ жесткое, поэтому $F$ является исключительным векторньм расслоением на поверхности Энриквеса $S$. Ничего не известно о стабильности расслоений $\widehat{E}$ и $F$. Эта конструкция порождает много исключительных векторных расслоений, так как известен способ получения всех исключительных векторных расслоений на гладкой квадрике $Q$.

Аналогично можно рассмотреть квартику $X$ в $\mathbb{P}^{3}$, заданную уравнением $z_{0}^{4}+z_{1}^{4}-$ $z_{2}^{4}-z_{3}^{4}$. Это гладкая К3-поверхность (см. [8]). Пусть $T$ - автоморфизм пространства $\mathbb{P}^{3}$ вида

$$
T:\left(z_{0}, z_{1}, z_{2}, z_{3}\right) \rightarrow\left(z_{0}, \sqrt{-1} z_{1},-z_{2},-\sqrt{-1} z_{3}\right) .
$$

У этого автоморфизма четыре неподвижные точки в $\mathbb{P}^{3}$, ни одна из которых не лежит на поверхности $X$; у автоморфизма $T^{2}$ две неподвижные прямые

$$
l_{1}=\left(z_{0}=z_{2}=0\right), \quad l_{2}=\left(z_{1}=z_{3}=0\right) .
$$

Прямые $l_{1}$ и $l_{2}$ пересекают поверхность $X$ в восьми точках $p_{1}, \ldots, p_{8}$. Рассмотрим раздутие $\bar{X} \rightarrow X$ поверхности $X$ в этих восьми точках. Обозначим через $\bar{T}$ индуцированньй автоморфизм на $\bar{X}$. Оказьвается, что поверхность $X^{\prime}=\bar{X} /\left\{\bar{T}^{2 n}\right\}$ является К3-поверхностью и $\bar{T}$ действует на $X^{\prime}$ как инволюция без неподвижных точек. Поэтому $X^{\prime} / \bar{T}$ является поверхностью Энриквеса $S$. Таким образом, по каждому исключительному векторному расслоению на $\mathbb{P}^{3}$ построено исключительное векторное расслоение на $S$; действительно, в силу того, что каждое исключительное расслоение на $\mathbb{P}^{3}$ однородно, оно является и $T$-инвариантньм. Конечно, полученное таким образом векторное расслоение на $X^{\prime}$ исключительно и его образ - тоже исключительное векторное расслоение. Эта конструкция приводит к большому числу исключительных векторных расслоений на $S$, которые можно описать, зная исключительные векторные расслоения на $\mathbb{P}^{3}$.

Теперь рассмотрим возможность построения исключительных наборов на поверхности Энриквеса. Напомним, что по определению набор векторных расслоений $E_{1}, \ldots, E_{n}$ является исключительным, если $\operatorname{Ext}^{i}\left(E_{k}, E_{j}\right)=0$ для всех $i$ и $k>j$. В частности, $\chi\left(E_{k}, E_{j}\right)=0$. Но на поверхности Энриквеса $\chi(E, F)=\chi(F, E)$ для любых пучков $E$ и $F$. Поэтому для исключительного набора на поверхности Энриквеса должны вьполняться условия

$$
\operatorname{Ext}^{i}\left(E_{k}, E_{j}\right)=0 \quad \forall i \text { и } k>j, \quad \chi\left(E_{a}, E_{b}\right)=0 \text { при } a \neq b .
$$

На общей поверхности Энриквеса существует исключительный набор из десяти расслоений. Известно, что на общей поверхности Энриквеса есть десять различных эллиптических пучков, скажем $\left|2 F_{1}\right|, \ldots,\left|2 F_{10}\right|$ (см. [4]). Легко видеть, что $\operatorname{Ext}^{i}\left(F_{k}, F_{j}\right)=0$ 
$\forall i$ и $k \neq j$, поэтому набор $F_{1}, F_{2}, \ldots, F_{10}$ исключительный. Было бы очень интересно описать ортогональную категорию в производной категории $D(S)$ пучков на поверхности Энриквеса $S$. В этой ортогональной категории должно быть всего два независимых элемента.

6. Модулярные операции. Существуют естественные модулярные отображения из одного пространства модулей в другое, определяющие изоморфизм касательных пространств к этим пространствам модулей. $\mathrm{K}$ ним относятся отображения $E \leftrightarrow E^{*}$, $E \leftrightarrow E \otimes D$, где $D$ - линейное расслоение. На поверхности Энриквеса существует очень интересная модулярная операция, которую назьвают отражснием. Эта операция аналогична отражению на К3-поверхностях (см. [9, 4.10, 4.11]). На решетке Мукая это отражение действует следующим образом:

$$
v=(r, D, s) \leftrightarrow R(v)=\widehat{v}=\left(2 s, D+\left(s+\frac{r}{2}\right) K_{S}, \frac{r}{2}\right) .
$$

Заметим, что $v^{2}=2 r s-D^{2}=\widehat{v}^{2}$.

Теперь опишем отражение в терминах пучков. Предположим, что пучок $E$ без кручения порожден своими сечениями и

$$
v(E)=v=(r, D, s), \quad \chi(E \otimes K)=h^{0}(E \otimes K), \quad h^{1}(E \otimes K)=h^{1}(E)=0 .
$$

Заметим, что отсюда следует $h^{2}(E \otimes K)=h^{2}(E)=0, h^{0}(E)=\chi(E)=\chi(E \otimes K)$. Если, например, подкрутить произвольное стабильное расслоение на достаточно большой очень обильньй дивизор, то необходимое условие будет вьполнено. Рассмотрим следующую точную последовательность:

$$
0 \rightarrow \bar{E}^{*} \rightarrow H^{0}(E) \otimes O_{S} \stackrel{\text { ev }}{\rightarrow} E \rightarrow 0
$$

где еv: $H^{0}(E) \otimes O_{S} \rightarrow E \rightarrow 0$ является каноническим отображением свертки (по предположению сюръективньм). Для удобства введем обозначения $H=H^{0}(E), h=\operatorname{dim} H$ и рассмотрим двойственную последовательность

$$
0 \rightarrow E^{*} \rightarrow H^{*} \otimes O_{S} \rightarrow \bar{E} \rightarrow 0
$$

Из наших условий и двойственности Серра вытекает, что

$$
h^{1}(\bar{E})=h^{2}\left(E^{*}\right)=h^{0}(E \otimes K)=\chi(E \otimes K)=h .
$$

Рассмотрим последовательность

$$
0 \rightarrow H^{1}(\bar{E}) \otimes K_{S} \rightarrow \widehat{E} \rightarrow \bar{E} \rightarrow 0
$$

где $\widehat{E}$ задается универсальным коциклом id $\in \operatorname{Ext}^{1}\left(\bar{E}, H^{1}(\bar{E})\right)=\operatorname{End}\left(H^{1}(\bar{E})\right)$. Положим $R(E)=\widehat{E}$. Из последовательностей $(1)$ и $(3)$, наших предположений и двойственности Серра непосредственно вытекает следующее утверждение. 
ПРЕДЛОЖЕНИЕ 2. Предположим, что пучок Е удовлетворяет изложенным выше предполохениям. Тогда пучки $\bar{E}$ и $\widehat{E}$ из последовательностей (1) $и$ (3) обладают следующими свойствами:

1) $\bar{E}$ глобально порожден своими сечениями;

2) $\chi(\bar{E})=0, h^{0}(\bar{E})=h=h^{1}(\bar{E}), h^{2}(\bar{E})=0$;

3) $\chi(\bar{E} \otimes K)=0, h^{i}\left(\bar{E} \otimes K_{S}\right)=0 \quad \forall i>0$.

4) $v(\widehat{E})=\widehat{v}=R(v)=\left(2 s, D+(s+r / 2) K_{S}, r / 2\right) u h^{0}(\widehat{E})=h^{0}(\widehat{E} \otimes K)=h$, $h^{i}(\widehat{E})=h^{i}(\widehat{E} \otimes K)=0 \quad \forall i>0 ;$

5) $\operatorname{Hom}(E, E)=\operatorname{Hom}(\bar{E}, \bar{E})=\operatorname{Hom}(\widehat{E}, \widehat{E}), \operatorname{Ext}^{2}(\bar{E}, \bar{E})=0$;

6) $\operatorname{rank}(E)=\operatorname{rank}(\widehat{E})(\bmod 2) ;$ более того, если $\operatorname{rank}(E)=2 k+1$ и оба пучка $E$ $u \widehat{E} H$-стабильны, mо $\operatorname{Ext}^{2}(\widehat{E}, \widehat{E})=\operatorname{Ext}^{2}(E, E)=0, \operatorname{Ext}^{1}(\widehat{E}, \widehat{E})=\operatorname{Ext}^{1}(E, E)$.

ДокАЗАТЕльство. 1) Пучок $H^{*} \otimes O_{S}$ в середине последовательности (2) глобально порожден своими сечениями, поэтому то же справедливо и для пучка $\bar{E}$.

2) Длинная точная последовательность когомологий, отвечающая последовательности $(2)$, дает $h^{0}(\bar{E})=h$, так как из двойственности Серра вытекает, что $h^{0}\left(E^{*}\right)=$ $h^{2}(E \otimes K)=0$ и $h^{1}\left(E^{*}\right)=h^{1}(E \otimes K)=0$. Кроме того, как уже отмечалось, $h^{2}(\bar{E})=0$ и $h^{1}(\bar{E})=h$. Поэтому $\chi(\bar{E})=0$.

3) Рассмотрим последовательность (2), подкрученную на $K$ :

$$
0 \rightarrow E^{*} \otimes K \rightarrow H^{*} \otimes K_{S} \rightarrow \bar{E} \otimes K \rightarrow 0
$$

Аналогично, длинная точная когомологическая последовательность дает $h^{0}(\bar{E} \otimes K)=$ $h^{1}\left(E^{*} \otimes K\right)=h^{1}(E)$ и $h^{1}(\bar{E} \otimes K)=0$, так как $h^{2}\left(E^{*} \otimes K\right)=h^{0}(E)=h=h^{2}\left(H^{*} \otimes K\right)$; $h^{2}(\bar{E} \otimes K)=h^{0}\left(\bar{E}^{*}\right)$ (в силу последовательности $\left.(1)\right)$.

4) Несложное вычисление показывает, что $v(\widehat{E})=\widehat{v}=\left(2 s, c_{1}(E)+(s+r / 2) K_{S}, r / 2\right)$. Так как последовательность (3) представляет собой универсальное расширение, то $h^{1}(\widehat{E})=h^{2}(\widehat{E})=0$, поэтому по теореме Римана-Роха $h^{0}(\widehat{E})=h$. Подкрутив $(3)$ на $K$ и воспользовавшись свойствами пучка $\bar{E} \otimes K$, легко получаем, что $h^{0}(\widehat{E} \otimes K)=h$, $h^{1}(\widehat{E} \otimes K)=h^{2}(\widehat{E} \otimes K)=0$.

5) Применив $\operatorname{Hom}(\bar{E}, *)$ к $(2)$, получим длинную точную последовательность

$$
\begin{aligned}
0 & \rightarrow \operatorname{Ext}^{0}\left(\bar{E}, E^{*}\right) \rightarrow \operatorname{Ext}^{0}\left(\bar{E}, H^{*} \otimes O_{S}\right) \rightarrow \operatorname{Ext}^{0}(\bar{E}, \bar{E}) \\
& \rightarrow \operatorname{Ext}^{1}\left(\bar{E}, E^{*}\right) \rightarrow \operatorname{Ext}^{1}\left(\bar{E}, H^{*} \otimes O_{S}\right) \rightarrow \operatorname{Ext}^{1}(\bar{E}, \bar{E}) \\
& \rightarrow \operatorname{Ext}^{2}\left(\bar{E}, E^{*}\right) \rightarrow \operatorname{Ext}^{2}\left(\bar{E}, H^{*} \otimes O_{S}\right) \rightarrow \operatorname{Ext}^{2}(\bar{E}, \bar{E}) \rightarrow 0 .
\end{aligned}
$$

Ввиду двойственности Серра и утверждения 3) средние члены строчек имеют вид $H^{i}(\bar{E} \otimes K) \otimes H^{*}=0 . \quad$ Поэтому $\operatorname{Ext}^{1}\left(\bar{E}, E^{*}\right)=\operatorname{Ext}^{0}(\bar{E}, \bar{E}), \operatorname{Ext}^{2}(\bar{E}, \bar{E})=0$. Аналогично, применяя $\operatorname{Hom}\left(*, E^{*}\right)$ к $(2)$, получим

$$
\begin{aligned}
0 & \rightarrow \operatorname{Ext}^{0}\left(\bar{E}, E^{*}\right) \rightarrow \operatorname{Ext}^{0}\left(H^{*} \otimes O_{S}, E^{*}\right) \rightarrow \operatorname{Ext}^{0}\left(E^{*}, E^{*}\right) \\
& \rightarrow \operatorname{Ext}^{1}\left(\bar{E}, E^{*}\right) \rightarrow \operatorname{Ext}^{1}\left(H^{*} \otimes O_{S}, E^{*}\right) \rightarrow \operatorname{Ext}^{1}\left(E^{*}, E^{*}\right) \rightarrow .
\end{aligned}
$$

Из наших предположений и двойственности Серра вытекает, что средние элементы строчек имеют вид

$$
\operatorname{Ext}^{0}\left(H^{*} \otimes O_{S}, E^{*}\right)=H^{2}(E \otimes K)=0=\operatorname{Ext}^{1}\left(H^{*} \otimes O_{S}, E^{*}\right)=H^{1}(E \otimes K) .
$$


Таким образом, $\operatorname{Hom}\left(E^{*}, E^{*}\right)=\operatorname{Hom}(E, E)=\operatorname{Hom}(\bar{E}, \bar{E})$. Применение $\operatorname{Hom}(\widehat{E}, *)$ к $(3)$ дает

$$
\begin{aligned}
& 0 \rightarrow \operatorname{Ext}^{0}(\widehat{E}, H \otimes K) \rightarrow \operatorname{Ext}^{0}(\widehat{E}, \widehat{E}) \rightarrow \operatorname{Ext}^{0}(\widehat{E}, \bar{E}) \rightarrow \\
& 0 \rightarrow \operatorname{Ext}^{1}(\widehat{E}, H \otimes K) \rightarrow \operatorname{Ext}^{1}(\widehat{E}, \widehat{E}) \rightarrow \operatorname{Ext}^{1}(\widehat{E}, \bar{E}) \rightarrow .
\end{aligned}
$$

В силу того, что $\operatorname{Ext}^{0}(\widehat{E}, H \otimes K)=H^{2}(\widehat{E}) \otimes H=0$, получаем $\operatorname{Ext}^{0}(\widehat{E}, \widehat{E})=\operatorname{Ext}^{0}(\widehat{E}, \bar{E})$. И, аналогично, применяя $\operatorname{Hom}(*, \bar{E})$, имеем $\operatorname{Ext}^{0}(\widehat{E}, \widehat{E})=\operatorname{Ext}^{0}(\bar{E}, \bar{E})$.

$6) \operatorname{Ecли~} \operatorname{rank}(E)$ нечетен, то $s(E) \in \frac{1}{2} \mathbb{Z}$, но $s(E) \notin \mathbb{Z}$, поэтому число $\operatorname{rank}(\widehat{E})=2 s(E)$ тоже нечетно. Если же $\operatorname{rank}(E)$ четен, то $s(E) \in \mathbb{Z}$, поэтому $\operatorname{rank}(\widehat{E})=2 s(E)$ тоже четен. Рассмотрим произвольньй ненулевой элемент $\varphi$ в $\operatorname{Ext}^{2}(E, E)=\operatorname{Ext}^{0}(E, E \otimes K)^{*}$. Ввиду стабильности элемент $\varphi$ должен быть изоморфизмом, но тогда $\operatorname{det} \varphi$ представляет собой ненулевой элемент канонического класса. Это противоречие показывает, что $\operatorname{Ext}^{2}(E, E)=0$ и $\operatorname{Ext}^{2}(\widehat{E}, \widehat{E})=0$. Из того, что $v^{2}=\widehat{v}^{2}$, следует $\operatorname{Ext}^{1}(E, E)=\operatorname{Ext}^{1}(\widehat{E}, \widehat{E})$.

В следующей ситуации мы можем отражение обратить. Рассмотрим пучок $F$ и предположим, что имеет место такая точная последовательность:

$$
0 \rightarrow H^{0}\left(F \otimes K_{S}\right) \otimes K_{S} \stackrel{\text { ev }}{\rightarrow} F \rightarrow \bar{F} \rightarrow 0
$$

где ev : $H^{0}\left(F \otimes K_{S}\right) \otimes K_{S} \rightarrow F-$ каноническая свертка. Предположим, что $\bar{F}$ - глобально порожденньй пучок без кручения. Тогда имеет место следующая точная последовательность:

$$
0 \rightarrow \widehat{F}^{*} \rightarrow H^{0}(\bar{F}) \otimes O_{S} \stackrel{\text { ev }}{\rightarrow} \bar{F} \rightarrow 0
$$

Положим $R(F)=\widehat{F}$. При этих предположениях справедлив аналогичньй результат.

ПРЕДЛОЖЕНИЕ 3. Предположим, что пучок F удовлетворяет указанным предположениям. Тогда для пучков $\bar{F}$ и $\widehat{F}$ из последовательностей (4) и (5) справедливы следуюшие утверждения:

1) $\chi(\bar{F})=0, h^{0}(\bar{F})=h=h^{1}(\bar{F}), h^{2}(\bar{F})=0$;

2) $\chi(\bar{F} \otimes K)=0, h^{i}\left(\bar{F} \otimes K_{S}\right)=0 \quad \forall i>0$;

3) $v(\widehat{F})=\widehat{v}=\left(2 s, D+(s+r / 2) K_{S}, r / 2\right) u h^{0}(\widehat{F})=h^{0}(\widehat{F} \otimes K)=h, h^{i}(\widehat{F})=$ $h^{i}(\widehat{F} \otimes K)=0 \quad \forall i>0 ;$

4) $\operatorname{Hom}(F, F)=\operatorname{Hom}(\bar{F}, \bar{F})=\operatorname{Hom}(\widehat{F}, \widehat{F}), \operatorname{Ext}^{2}(\bar{F}, \bar{F})=0$;

5) $\operatorname{rank}(F)=\operatorname{rank}(\widehat{F})(\bmod 2) ;$ более mого, если $\operatorname{rank}(F)=2 k+1$ и оба пучка $F$ $u \widehat{F} H-c m a б и л ь н ы, ~ m o \operatorname{Ext}^{2}(\widehat{F}, \widehat{F})=\operatorname{Ext}^{2}(F, F)=0, \operatorname{Ext}^{1}(\widehat{F}, \widehat{F})=\operatorname{Ext}^{1}(F, F)$.

Заметим, что из предложений 2 и 3 вытекает, что $R(R(E))=E$.

ЗАмЕчАниЕ. Отражение всегда существует в производной категории пучков на поверхности $S$. При этом неважно, сюръективно или инъективно отображение свертки $H^{0}(F) \otimes O_{S} \stackrel{\text { ev }}{\rightarrow} F$.

Теперь приведем несколько примеров отражений. 
ПримеР 1. Пусть $C$ - гладкая кривая на $S, A$ - глобально порожденньй дивизор на $C$, обладаюший следуюшими свойствами: $h^{1}\left(O_{C}(A)\right)=h^{1}\left(O_{C}\left(A \otimes K_{S}\right)\right)=0$. Рассмотрим точную последовательность

$$
0 \rightarrow E(C, A)^{*} \rightarrow H^{0}\left(O_{C}(A)\right) \otimes O_{S} \stackrel{\text { ev }}{\rightarrow} O_{C}(A) \rightarrow 0 .
$$

Двойственная к (6) последовательность имеет вид

$$
0 \rightarrow H^{0}(A)^{*} \otimes O_{S} \rightarrow E(C, A) \rightarrow O_{C}(C) \otimes A^{*} \rightarrow 0 .
$$

В силу наших предположений, этой последовательности и двойственности Серра на кривой $C$ имеем $h^{1}(E(C, A))=h^{1}\left(O_{C}(C-A)\right)=h^{0}\left(O_{C}(A)\right)$, откуда вытекает последовательность

$$
0 \rightarrow H^{1}(E(C, A)) \otimes K_{S} \rightarrow \widehat{E} \rightarrow E(C, A) \rightarrow 0
$$

Поэтому $R\left(O_{C}(A)\right)=\widehat{E}$.

Если $C$ - $(-2)$-кривая и $A=O_{C}$, то $R\left(O_{C}\right)=F$ - экстремальное векторное расслоение ранга 2, т.е. $\operatorname{Ext}^{0}(F, F)=\operatorname{Ext}^{2}(F, F)=\mathbb{C}, \operatorname{Ext}^{1}(F, F)=0$.

ПримеР 2. Если $|2 F|,|2 G|$ - два эллиптических пучка на $S$, то у пучка $|F+G|$ две различные базисные точки $x$ и $y$. Стандартная последовательность

$$
0 \rightarrow J_{x+y}(F+G) \rightarrow O(F+G) \rightarrow O_{x+y}(F+G) \rightarrow 0
$$

показывает, что $h^{1}\left(J_{x+y}(F+G)\right)=2$. В результате получим следующее трехмерное расслоение $E$, определенное универсальньм коциклом id $\in \operatorname{End}\left(H^{1}\left(J_{x+y}(F+G)\right)\right)$ :

$$
0 \rightarrow H^{1}\left(J_{x+y}(F+G)\right) \otimes K_{S} \rightarrow E \rightarrow J_{x+y}(F+G) \rightarrow 0 .
$$

Согласно [9, леммы $1.1,1.2] E$ - простое расслоение. Несложное вычисление показывает, что расслоение $E$ исключительное. Кроме того, из предложения 3 вытекает, что $R(E)=O(F+G)$. Заметим, что расслоение $O(F+G)$ не порождено сечением глобально, поэтому к нему нельзя применить предложение 2 и получить $R(O(F+G))$, но это можно проделать в производной категории. Так как $R(E)=O(F+G)$, то также $R(O(F+G))=E$.

ПримеР 3. Дивизор $O(a F+b G)$ при $a \geqslant b \geqslant 2$ на общей поверхности Энриквеса обилен. Поэтому $R(O(a F+b G))$ - исключительное векторное расслоение ранга $2 a b+1$. 


\section{СПИСОК ЦИТИРОВАННОЙ ЛИТЕРАТУРЫ}

[1] Kim H. Exceptional bundles on nodal Enriques surfaces. Preprint. Bayreuth, 1991.

[2] Kim H. Exceptional bundles and moduli spaces of stable vector bundles on Enriques surfaces. Preprint. Bayreuth, 1991.

[3] Naie D. Special rank two vector bundles over Enriques surfaces. Preprint. Paris: Université de Paris-Sud, 1993.

[4] Cossec F., Dolgachev I. Enriques Surfaces 1. Basel: Birkhäuser, 1989.

[5] Кулешов С. А. Теорема существования исключительных расслоений на поверхностях типа К3 // Изв. АН СССР. Сер. матем. 1989. V. 53. № 2. Р. 363-378.

[6] Mukai S. On the moduli space of bundles on K3 surfaces. I // Vector bundles on algebraic varieties. Bombay: Tata Inst. Fund. Res., 1987. P. 341-413.

[7] Bart W., Peter C., Van de Ven A. Compact complex surfaces. Berlin-Heidelberg-New York: Springer Verlag, 1984.

[8] Гриффитс Ф., Харрис Дж. Принципы алгебраической геометрии. М.: Мир, 1982.

[9] Tyurin A. N. Cycles, curves and vector bundles on algebraic surfaces // Duke Math. J. 1987. V. 54. P. 1-26. 